\title{
ANALYSIS OF AN ATM MULTIPLEXER WITH PRIORITIZED SERVICE
}

\author{
J. F. Meyer \\ EECS Department, The University of Michigan \\ Ann Arbor, Michigan, USA
}

\author{
S. Montagna, R. Paglino, and A. Puglisi \\ Central Research Laboratories, Italtel S.p.A. \\ Milan, Italy
}

\begin{abstract}
We examine an ATM multiplexer that is able to support two classes of service having qualities specified at the cell level. The first is a Guaranteed Bandwidth (GB) service which ensures very small cell loss probabilities and cell delays. The amount of statistical multiplexing permitted for such connections is therefore quite limited. The second is one suited to non-real-time Variable Bit Rate (VBRnr) sources where much longer delays can be tolerated. In this case, larger buffers and higher levels of statistical multiplexing can be exploited to increase the utilization of the output link. To accommodate these differences, we consider a multiplexer with two buffers, one for each service class, where the GB connections have service priority. Specifically, the low-priority VBRnr traffic is served only when there are no cells in the buffer for the GB connections. Both types of traffic are modeled by On/Off sources. The principal aim of the investigation is to analyze the steady-state behavior of the low-priority buffer with respect to two hypotheses concerning the guaranteed traffic. The first presumes that the GB connections are allocated according to their peak bit rates; in this case, the analysis is exact. The second hypothesis permits partial statistical multiplexing of the GB traffic; here, the exact method is extended so as to provide an approximate solution. A simpler model is then considered for this purpose, which suffices if the low-priority buffer is sufficiently large. In conclusion, some attention is devoted to the transient behavior of this buffer, where we find that congestion probabilities can exceed those experienced under steady-state conditions.
\end{abstract}

The original version of this chapter was revised: The copyright line was incorrect. This has been corrected. The Erratum to this chapter is available at DOI: 10.1007/978-0-387-35353-1_28 
Keywords: ATM, multiplexer, service priority, analysis

\section{INTRODUCTION}

Broadband multiservice networks are intended to provide a variety of voice, data, and video services, where their corresponding requirements can differ considerably. For example, the loss of an ATM cell is quite acceptable for a voice connection while it may cause the retransmission of a large quantity of cells in the case of a data transfer. Accordingly, the ability of a network to discriminate between cells belonging to different service classes is advantageous, permitting its adaptation to class-specific needs. In particular, this can avoid inefficiencies associated with serving connections uniformly according to the most stringent requirements, thereby increasing network utilization.

Generally, the extent to which a common broadband transmission medium can support multiple services depends essentially on performance considerations. During the past decade, a great deal of attention has been devoted to characterizing such services in terms of quality requirements and, in turn, developing efficient means of integration that can guarantee specified service qualities (see Gopal et al., 1992; Bonomi and Fendick, 1995, for example). More sophisticated queueing disciplines, as an alternative to traditional FIFO scheduling, have also been investigated for this purpose, particularly those involving some form of priority mechanism F.Bonomi et al., 1990; Kroner et al., 1991; Zhang, 1993; Meyer et al., 1993.

One of the important consequences of this effort has been the standardization of a non-real-time Variable Bit Rate (VBRnr) service ATM Forum, 1996. This service class, originally developed for data transfer, has very strict loss-probability requirements but has no constraints with regard to transmission delays. Although the latter permits large buffers and good bandwidth utilization, if network resources are to be shared with Guaranteed Bandwidth (GB) services (those with strict delay requirements), the GB connections require special treatment. An obvious solution in this regard is a service priority mechanism that favors GB sources without compromising the loss requirements for the VBRnr sources. Specifically, the investigation that follows considers the use of dedicated buffers for this purpose, one for each service class, where the VBRnr traffic is served only when there are no cells in the buffer for the GB connections.

Section 2 examines an architecture of this kind, with the assumption of a single server (the channel) and two finite-capacity buffers. As sug- 
gested by the above remarks, the capacity of the low-priority (VBRnr) buffer will be typically be much larger than that employed for the highpriority GB traffic. The analysis concerns the occupancy distribution of the low-priority buffer with respect to two alternative assumptions concerning the allocation of high-priority sources. In the first case we assume that there is no statistical multiplexing of the high-priority GB traffic, i.e., these connections are admitted until the sum of their peak bit rates is equal to the channel capacity. Under the second hypothesis, we allow partial statistical multiplexing of the GB traffic but require that the congestion probability at the burst level be less than a target loss probability of $10^{-10}$ (see Rasmussen et al., 1991, for example). Solution algorithms, based on an appropriate stochastic model, are then developed for each hypothesis, where the first admits to an exact analysis and the second to an approximate solution.

Results of applying these algorithms are then presented in Section 3, indicating the effects of various On/Off traffic assumptions on the distribution of the VBRnr buffer. In the case of the approximate solution method, comparisons are made with simulation data. Comparisons are also made with distributions obtained from a multiplexer without priority for both classes of traffic. The results show that, as the capacity of the VBRnr buffer increases, these distributions approach those given by the algorithms of Section 2. Accordingly, if the VBRnr buffer is sufficiently large, one can use this simpler approach to approximate its distribution. In particular, this permits the use of approximate methods such as those described in Sohraby, 1992; Acampora and Zhang, 1992. Section 3 concludes with brief study of the transient behavior of the low-priority buffer, where we find that congestion probabilities can exceed those experienced under steady-state conditions. The final section (Section 4) then summarizes the approach and main results of the entire investigation.

\section{MATHEMATICAL MODEL AND ANALYSIS}

Consider the system described in Figure 9.1, where buffers $B_{0}$ and $B_{1}$ have finite capacities $Q_{0}$ and $Q_{1}$, respectively, and are served by a common channel with capacity $C$. Buffer $B_{0}$ is reserved for GB connections which have very low delay requirements and have priority in accessing the channel relative to cells that enter $B_{1}$. The latter are presumed to derive from VBRnr connections, which have no delay requirements and are served only when buffer $B_{0}$ is empty. In keeping with the notation 
for the buffers, we refer to the GB and VBRnr traffic as being type 0 and type 1 , respectively. The service discipline for both buffers is FIFO.

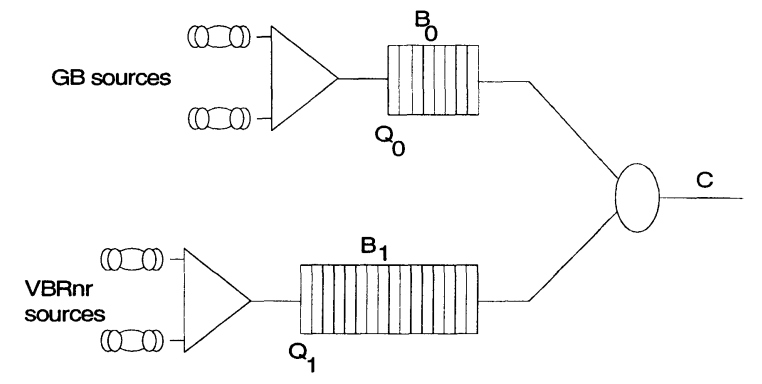

Figure 9.1 Service priority multiplexer.

More precisely, traffic of type $j(j=0,1)$ is assumed to be a set of $N_{j}$ homogeneous On/Off sources, where each individual source is modeled by a 2 -state Markov process. If a type- $j$ source is active (the On state), cells arrive periodically during time slots that are separated by some specified integer $R_{j} \geq 1$, where the values of $R_{0}$ and $R_{1}$ may differ. In other words, an active type- $j$ source transmits cells at a peak rate equal to $C / R_{j}$. If a source of either type is idle (the Off state) then no cells are transmitted. Accordingly, if we let $T=\{0,1,2, \ldots\}$ denote the model's (discrete) time base, where a time instant $t \in T$ is interpreted as $t$ th time slot, then cells from a type- $j$ source can arrive only during slots $t$ that are non-negative integer multiples of $R_{j}$. Hence, for each traffic class, all $N_{j}$ of the type- $j$ connections are synchronized in the sense that cell arrivals and source-state transitions can occur only at times

$$
t=s R_{j}, s \in\{0,1,2, \ldots\} .
$$

Although alternative assumptions could made with regard to the relative phasing of sources (including the possibility of random phasing), the justification of any such assumption is not obvious. On the other hand, tight synchronization of the kind assumed above, although not realistic from a practical viewpoint, results in worst-case buffer congestion and cell delays. Our subsequent analysis is therefore conservative and, in this sense, the constraints imposed by (9.1) appear to be reasonable.

Due to the presumed On/Off nature of individual sources, the number of consecutive periods (each of length $R_{j}$ ) during which a type- $j$ source remains active is geometrically distributed with mean $L_{j}$. If further, we let $I_{j}$ denote the mean value of the Off time (which likewise has a geometric distribution and, again, is quantified in units equal to $R_{j}$ ) 
then the activity $\rho_{j}$ of a type- $j$ source is determined by the relation $\rho_{j}=L_{j} /\left(I_{j}+L_{j}\right)$. Alternatively, if we choose to specify $\rho_{j}$ and $L_{j}$ then the mean Off time $I_{j}$ is uniquely determined. The latter choice, together with our synchronization assumption, implies that the type- $j$ traffic entering buffer $B_{j}(j=0,1)$ is completely characterized by the values of four parameters, namely $N_{j}, R_{j}, \rho_{j}$, and $L_{j}$.

Because of the stringent delay requirement for type-0 (GB) traffic, the queueing of such cells must be kept to a minimum. This requires a specific control on the number $N_{0}$ of connections permitted for this traffic class. Accordingly, the investigation that follows considers each of two alternative hypotheses regarding the allocation of type- 0 connections.

H1: Peak Allocation. In this case, the number of high-priority connections is restricted such that it does not exceed the period $R_{0}$, i.e., $N_{0} \leq R_{0}$. Since the sources are synchronized (9.1), at each potential arrival time $s R_{0}$, there are at most $N_{0}$ cell arrivals of type 0 . Hence, one can easily verify the following observation. If the capacity of buffer $B_{0}$ is at least $N_{0}$ then the number of cells $q_{t}$ in $B_{0}$ at the end of slot $t$, given that $m$ type- 0 sources are active, is

$$
q_{t}=\max \left\{m-\left(t \bmod R_{0}\right), 0\right\} .
$$

Taking the capacity of $B_{0}$ to be equal to the number of type- 0 sources (i.e., $Q_{0}=N_{0}$ ), the above implies that $q_{t} \leq Q_{0}$, for all $t \in T$. Hence, no high-priority cells are lost under hypothesis H1.

H2: Cell-level Statistical Multiplexing. In this case, we suppose that $N_{0}>R_{0}$ while imposing the following restriction. Let $X_{0}$ be the random variable whose value is the number of active type0 sources under steady-state conditions. Assuming further that $Q_{0}=R_{0}$, we then want to insure that $P\left[X_{0}>R_{0}\right]<P_{\text {loss }}$, where $P_{\text {loss }}$ is the target loss probability. The value of $P\left[X_{0}>R_{0}\right]$ that satisfies this requirement can be determined from the steady-state distribution of the (composite) source model, where this value increases as $N_{0}$ becomes larger.

Under either of the above hypotheses, we note that the maximum value of $N_{0}$ does not depend on $L_{0}$. Moreover, the occupancy distribution of buffer $B_{0}$ is independent of the low-priority (type-1) traffic and, hence, is easily obtained. Accordingly, the more interesting distribution is that of buffer $B_{1}$, since it can reveal the advantages of statistical multiplexing with respect to the remaining available bandwidth. In what follows, we show how this distribution may be evaluated without having to account for the behavior of $B_{0}$. As mentioned in Section 1, under 
hypothesis $\mathrm{H} 1$, this analysis is exact; if $\mathrm{H} 2$ is presumed, the resulting solution is approximate.

For these purposes, we define a finite-state stochastic process with respect to the time base $T=\{0,1,2, \ldots\}$ where, as noted earlier, an element $t \in T$ is interpreted as the time of the $t$ th slot. As such, these times also correspond to transmission instants on the output link. More precisely, unless both buffers are empty at the end of slot $t-1$, a cell is transmitted at the beginning of slot $t$. Following such a departure (if it exists), a type $j$ cell may arrive depending on (9.1) and whether a source of that type is active. The buffer occupancies are then observed at the end of the time slot. With these assumptions, a state of process can be described by a 3 -tuple $(q, m, n)$, where $q$ is the number of cells in $B_{1}, m$ is the number of active type- 0 sources, and $n$ is the number of active type- 1 sources. Accordingly, $0 \leq q \leq Q_{1}, 0 \leq m \leq N_{0}$, and $0 \leq n \leq N_{1}$. To describe the probabilistic nature of this process, for $t \in T$, we let $p_{t}(q, m, n)$ denote the probability of being in state $(q, m, n)$ at time $t$. The dependence of this probability on $t$, even when $t$ is large, is due to the fact that type- $j$ sources can change state only at times given by (9.1). Hence, except in the degenerate case where $R_{j}=1$ for both traffic classes, these times constitute a proper subset of $T$.

To determine the values of $p_{t}$, it is also necessary to know whether the server is able to accept a low-priority (type-1) service at time $t$. Because the occupancy of the high-priority buffer $B_{0}$ is not included as part of the model's state, such information must be inferred from the type- 0 source states. In the case of peak allocation (hypothesis $\mathrm{H} 1$ ), this is possible; indeed, in a given slot $t$ with the system in state $(q, m, n)$, the server can transmit a low-priority cell if and only if $B_{0}$ was empty at the end of the previous slot, i.e., $(t-1) \bmod R_{0} \geq m^{\prime}$, where $m^{\prime}$ is the number of active type- 0 sources at time $t-1$. On the other hand, if even a limited amount of statistical multiplexing is used to allocate the GB connections (hypothesis H2), this kind of inference is no longer possible. Nevertheless, via an approximate solution method that involves a modified type- 0 source model, we find that the same algorithm can be applied.

To describe this algorithm, let $A_{j}$ denote the transition matrix for the active sources of type $j(j=0,1)$ and let $a_{j}(k, \ell)$ denote its $k, \ell$ entry. In other words, $a_{j}(k, \ell)$ is the conditional probability that $\ell$ type- $j$ sources are active at a potential arrival time $s R_{j}$, given that $k$ were active at the previous potential arrival time $(s-1) R_{j}$ (where $0 \leq k, \ell \leq N_{j}$ and $s=1,2,3, \ldots)$. Since sources of a given type are synchronized, the formulas for these probabilities are identical to those derived for successiveslot transitions of combined On/Off sources (see Bonomi et al., 1992, for 
example). Accordingly, under hypothesis $\mathrm{H} 1$, the state probability at time $t$ can be expressed recursively as a function of the state probabilities at time $t-1$. More precisely, if we let $c(t)=(t-1) \bmod R_{0}$ then, for $q<Q_{1}$, we have the following case-by-case formulations of the stateoccupancy probabilities at time $t$.

Case 1: $t$ is an integer multiple of neither $R_{0}$ nor $R_{1}$. In this case, there are no arrivals and no change in the number of active sources. If $B_{1}$ is nonempty at time $t-1$ and the channel is available, the low-priority buffer has one less cell at time $t$. Accordingly, value of $p_{t}(q, m, n)$, can be formulated in terms of the previous slot's distribution as follows.

$$
p_{t}(q, m, n)= \begin{cases}p_{t-1}(q, m, n) & \text { if } c(t)<m \\ p_{t-1}(q, m, n)+p_{t-1}(q+1, m, n) & \text { if } c(t) \geq m \text { and } q=0 \\ p_{t-1}(q+1, m, n) & \text { if } c(t) \geq m \text { and } q>0\end{cases}
$$

Case 2: $t$ is an integer multiple of $R_{0}$ but not of $R_{1}$. Here, only GB sources can change state. The low-priority buffer $B_{1}$ can transmit a cell only if the previous number of high-priority arrivals was less than $R_{0}$. Hence, the recursive formulation in this case is

$$
p_{t}(q, m, n)= \begin{cases}\sum_{m^{\prime}=0}^{R_{0}-1} p_{t-1}\left(q+1, m^{\prime}, n\right) a_{0}\left(m^{\prime}, m\right) & \\ +\sum_{m^{\prime}=R_{0}}^{N_{0}} p_{t-1}\left(q, m^{\prime}, n\right) a_{0}\left(m^{\prime}, m\right) & \text { if } q>0 \\ \sum_{m_{0}-1}^{R^{\prime}=0} p_{t-1}\left(1, m^{\prime}, n\right) a_{0}\left(m^{\prime}, m\right) & \\ +\sum_{m^{\prime}=0}^{N_{0}} p_{t-1}\left(0, m^{\prime}, n\right) a_{0}\left(m^{\prime}, m\right) & \text { if } q=0\end{cases}
$$

where a sum is understood to have value 0 if the lower limit exceeds the upper limit.

Case 3: $t$ is an integer multiple of $R_{1}$ but not of $R_{0}$. In this case, low-priority sources can change state and we can have a corresponding number of arrivals entering the low-priority buffer.

$$
p_{t}(q, m, n)= \begin{cases}\sum_{n^{\prime}=0}^{N_{1}} p_{t-1}\left(q-n, m, n^{\prime}\right) a_{1}\left(n^{\prime}, n\right) & \text { if } c(t)<m \text { and } q \geq n \\ \sum_{q^{\prime}=0}^{1} \sum_{n^{\prime}=0}^{N_{1}} p_{t-1}\left(q^{\prime}, m, n^{\prime}\right) a_{1}\left(n^{\prime}, n\right) & \text { if } c(t) \geq m \text { and } q=n \\ \sum_{n^{\prime}=0}^{N_{1}} p_{t-1}\left(q-n+1, m, n^{\prime}\right) a_{1}\left(n^{\prime}, n\right) & \text { if } c(t) \geq m \text { and } q>n\end{cases}
$$


Case 4: $t$ is an integer multiple of both $R_{0}$ and $R_{1}$. This is the most complicated case since, during such a slot, cells may arrive at both buffers and source states may change for each type of traffic.

$$
p_{t}(q, m, n)= \begin{cases}\sum_{m^{\prime}=0}^{R_{0}-1} \sum_{n^{\prime}=0}^{N_{1}} p_{t-1}\left(q-n+1, m^{\prime}, n^{\prime}\right) a_{0}\left(m^{\prime}, m\right) a_{1}\left(n^{\prime}, n\right) & \\ +\sum_{m^{\prime}=R_{0}}^{N_{0}} \sum_{n^{\prime}=0}^{N_{1}} p_{t-1}\left(q-n, m^{\prime}, n^{\prime}\right) a_{0}\left(m^{\prime}, m\right) a_{1}\left(n^{\prime}, n\right) & \text { if } q>n \\ \sum_{q^{\prime}=0}^{1} \sum_{m^{\prime}=0}^{R_{0}-1} \sum_{n^{\prime}=0}^{N_{1}} p_{t-1}\left(q^{\prime}, m^{\prime}, n^{\prime}\right) a_{0}\left(m^{\prime}, m\right) a_{1}\left(n^{\prime}, n\right) & \\ +\sum_{m^{\prime}=R_{0}}^{N_{0}} \sum_{n^{\prime}=0}^{N_{1}} p_{t-1}\left(0, m^{\prime}, n^{\prime}\right) a_{0}\left(m^{\prime}, m\right) a_{1}\left(n^{\prime}, n\right) & \text { if } q=n \\ 0 & \text { if } q<n\end{cases}
$$

The equations for a full buffer $\left(q=Q_{1}\right)$ are a natural extension of the above relationships. Regarding Cases 1 and 3 , it should be noted that these are indeed possible, provided the period of the type- 0 sources is non-trivial $\left(R_{0}>1\right)$. Therefore, due to the dependence on time (via $c(t)$ ) that exists in these cases, the resulting model is generally a nonhomogeneous Markov process. Moreover, this process is periodic as a consequence of the source periods $R_{0}$ and $R_{1}$, precluding convergence of $p_{t}($ as $t \rightarrow \infty)$ to a stationary distribution On the other hand, once $t$ becomes sufficiently large, the distributions of $p_{t}$ will repeat periodically with a period equal to the least common multiple $1 \mathrm{~cm}\left(R_{0}, R_{1}\right)$ of periods $R_{0}$ and $R_{1}$. These distributions can thus be obtained by computing the distributions for each $t$, starting from an arbitrary distribution at time $t=0$, until their difference in two successive periods is negligible. More precisely, if we let $\ell(t)=t-1 \mathrm{~cm}\left(R_{0}, R_{1}\right)$, these computations are iterated until the equation

$$
\frac{\left|p_{t}(q, m, n)-p_{\ell(t)}(q, m, n)\right|}{p_{t}(q, m, n)}<\varepsilon
$$

is satisfied for each slot in the period and for each admissible state of the system. Typically, $\varepsilon$ is taken to be $10^{-5}$.

Let us now consider the alternative allocation hypothesis $\mathrm{H} 2$ which permits a limited amount of statistical multiplexing for the high-priority GB sources. Here we find that, by introducing a modified representation of these sources, the above formulas can be applied to obtain highly accurate approximate solutions. This "equivalent" type- 0 traffic model is constructed by reducing the number $N_{0}$ of high-priority sources to 
$R_{0}$ and increasing the activity of each source such that the load on the output link remains the same. More precisely, given that $N_{0}, R_{0}, \rho_{0}$, and $L_{0}$ are the parameters of the type- 0 sources (as defined above), for the modified model we consider parameters $N_{0}^{\prime}, R_{0}^{\prime}, \rho_{0}^{\prime}$, and $L_{0}^{\prime}$, where the first three are given by the equations

$$
N_{0}^{\prime}=R_{0}, \quad R_{0}^{\prime}=R_{0}, \text { and } \rho_{0}^{\prime}=\frac{N_{0} \rho_{0}}{N_{0}^{\prime}} .
$$

Accordingly, for given values of the original parameters, the link utilization due to type- 0 sources is the same for the equivalent representation (since $N_{0}^{\prime} \rho_{0}^{\prime}=N_{0} \rho_{0}$ ) and, moreover, the new parameter values are such that hypothesis H1 (peak allocation) is satisfied.

Concerning $L_{0}^{\prime}$, two possibilities are considered. The first is to let $L_{0}^{\prime}=L_{0}$; the second is to assign $L_{0}^{\prime}$ a value such that the asymptotic variance $v$ (see Jacobsen et al., 1990, for example) of the arrival process, defined by

$$
v=\lim _{t \rightarrow \infty} \frac{\operatorname{VAR}[N(0, t)]}{t}
$$

where $N(0, t)$ is the number of cells arriving in $[0, t]$, remains the same as that of the original traffic. In the section that follows we find that, even under the most unfavorable load conditions, these choices lead to distributions for buffer $B_{1}$ that are essentially identical.

\section{RESULTS}

We begin by examining results under hypothesis H1, i.e., the case where the GB sources require a bandwidth no greater than the channel capacity $C=150 \mathrm{Mbps}$. As noted earlier, under these conditions the algorithm yields an exact solution. Of initial interest is the possible influence of GB-traffic burstiness, as reflected by the mean burst length $L_{0}$, on the occupancy distribution of the low-priority buffer. Some preliminary observations in this regard are the following.

A) For GB (type-0) traffic, it follows from equation (9.2) that buffer $B_{0}$ is always empty prior to the entry of type- 0 cells. Accordingly, the occupancy distribution of $B_{0}$ is independent of the mean burst length $L_{0}$ and is equal to the distribution of the channel's busy period (when serving GB traffic only).

B) However, beyond a knowledge of this busy-period distribution (which can be obtained by analysis in the absence of VBRnr sources, i.e., $N_{1}=0$ ), it is important to note that there is correlation between the durations of two consecutive busy periods. This can be seen 
by observing the type- 0 cell departure process for two consecutive intervals of length $R_{0}$, assuming that the busy period duration of the first interval is equal to $\ell$ slots $\left(1 \leq \ell \leq N_{0}\right)$. The probability that the busy period in the second interval will have a duration of $m$ slots is then given by $a_{0}(\ell, m)$. This implies that, in general, the durations of consecutive busy periods are statistically dependent; moreover, the extent of this correlation is a function of the mean burst length $L_{0}$. Regarding the results in question, it is therefore reasonable to expect that the value of $L_{0}$ will have an impact on the distribution of the low-priority buffer.

This reasoning is borne out by Figures 9.2 and 9.3 which display the occupancy distribution of the low-priority buffer $B_{1}$. The capacity assumed for this buffer is $Q_{1}=400$ (note that these figures are truncated, ignoring occupancies which have extremely low probabilities). Recall that, under peak allocation (hypothesis $\mathrm{H} 1$ ), the capacity of the highpriority buffer $B_{0}$ is assumed to coincide with the number of high priority connections, i.e., $Q_{0}=N_{0}$; under hypothesis $\mathrm{H} 2$, we presume that $Q_{0}=$ $N_{0}^{\prime}=R_{0}$. With these choices, there are no type- 0 cell losses under $\mathrm{H} 1$ and negligible type- 0 losses under $\mathrm{H} 2$. Specifically, the type- 0 traffic assumed for Figure 9.2 consists of $N_{0}=3$ GB sources, each with a peak rate of $30 \mathrm{Mbps}$ (i.e., $R_{0}=5$ ) and activity $\rho_{0}=0.5$. For the sake of observing effects of burstiness, two values are considered for $L_{0}$, namely 20 and 100. The low-priority traffic assumed for this figure is given by the parameter values $N_{1}=10, R_{1}=15, \rho_{1}=0.5$, and $L_{1}=100$. In Figure 9.3, a different mix of traffic is considered, with more GB sources $\left(N_{0}=5\right)$, fewer VBRnr sources $\left(N_{1}=4\right)$, and a lower activity for both classes $\left(\rho_{0}=\rho_{1}=0.1\right)$. The periods are the same as for Figure $9.2\left(R_{0}=5, R_{1}=15\right)$ but the burstiness differs considerably, i.e., the comparison here is for values of $L_{0}$ equal to 5 and 200, with an assumed mean burst length of $L_{1}=10$ for the VBRnr traffic.

In both figures, we see that the burstiness of the high-priority sources has an appreciable influence on the occupancy distribution of the lowpriority buffer. Moreover, if the relative change in the value of $L_{0}$ is increased (e.g., the $40 \times$ increase considered in Figure 9.3 compared with the $5 \times$ change for Figure 9.2), this influence becomes more severe. Accordingly, these observations imply (assuming H1) that, although the distribution of buffer $B_{0}$ does not depend on the mean burst length $L_{0}$ of type- 0 traffic, the value of $L_{0}$ needs to be determined since it has an appreciable effect on the occupancy distribution of the low-priority buffer $B_{1}$. 


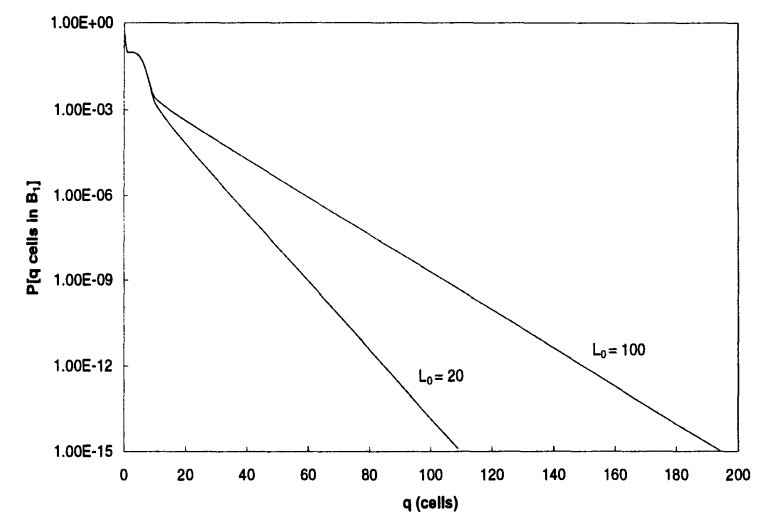

Figure 9.2 Influence of GB-source burstiness on buffer $B_{1} ; L_{0}=20,100$.

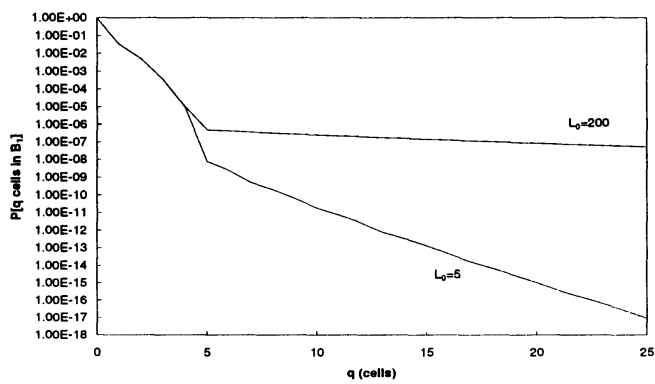

Figure 9.3 Influence of GB-source burstiness on buffer $B_{1} ; L_{0}=5,200$.

Turning now to results of using the approximate algorithm under hypothesis $\mathrm{H} 2$, for these instances we choose a small value for $\rho_{0}$, since it poses the worst condition for the approximation. This is due to the fact that, with higher loads, the number of sources that can be accommodated by the solution method proposed in Rasmussen et al., 1991 decreases rapidly toward the number permitted using peak allocation. Examining again the occupancy distribution of the low-priority buffer, the traffic parameter values assumed for Figure 9.4 are $N_{0}=45, R_{0}=15$, $\rho_{0}=0.05$, and $L_{0}=100$ for the high-priority traffic and $N_{1}=60$, 
$R_{1}=30, \rho_{1}=0.075$, and $L_{1}=200$ for the low-priority sources. Applying equations (9.3), the modified values for the first three type- 0 traffic parameters are therefore $N_{0}^{\prime}=15, R_{0}^{\prime}=15$, and $\rho_{0}^{\prime}=0.15$. Two choices are considered for $L_{0}^{\prime}$ according to the discussion at the end of Section 2, i.e., $L_{0}^{\prime}=100$ (equating it with $L_{0}$ ) and $L_{0}^{\prime}=111$ (by equating variances). As can be seen from Figure 9.4, the resulting approximate distributions for either choice are essentially the same. It can also be observed that, for buffer occupancies that are moderately large ( $\leq 15$ cells), the approximations agree almost exactly with a distribution obtained by simulating the exact model. For larger occupancies, the the approximate model unfortunately underestimates the probabilities obtained by simulation. Note, however, that this occurs in the low-probability region (tail) of the distribution where simulation results themselves are more susceptible to errors of estimation. In Figure 9.5, we consider type-0 sources with twice the mean burst length (when compared to Figure 9.4); the remaining parameter values are the same. Again, the choice of $L_{0}^{\prime}$ has very little effect on the solutions. Moreover, even with this amount of burstiness, the comparison with simulation data remains similar to what was observed in Figure 9.4.

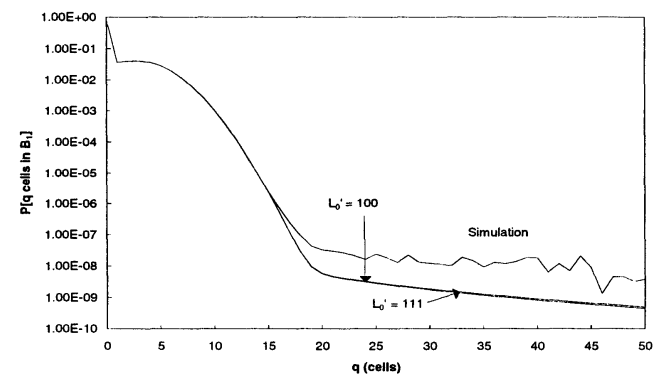

Figure 9.4 Validation of the approximate solution; $L_{0}=100$.

As noted earlier, algorithms such as these permit the evaluation of large buffers at the expense of long solution times. The latter is a serious problem, however, if such computations are to be part of a connection admission control (CAC) algorithm, calling for execution times in the order of tens of milliseconds. Prompted by this concern, we find (applying the solution algorithms described above) that the behavior 


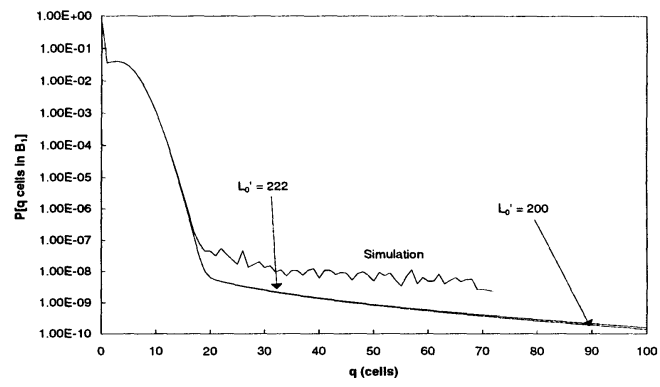

Figure 9.5 Validation of the approximate solution; $L_{0}=200$.

of the low-priority buffer is similar to that of a single-buffer multiplexer handling all the offered traffic (without prioritized service), provided the capacity of $B_{1}$ is sufficiently large. Given this condition is satisfied, it is therefore possible to employ particularly fast approximations such as those proposed in Sohraby, 1992; Acampora and Zhang, 1992.

To substantiate this claim, we first compare the low-priority buffer distributions (exact vs. the simpler 1-buffer model) when the GB-required bandwidth is equal to the channel capacity, thus satisfying hypothesis H1. (Other assumptions stated in $\mathrm{H} 1$ also apply here, e.g., the capacity $Q_{0}$ of buffer $B_{0}$ is equal to the number $N_{0}$ of type- 0 connections.) For traffic parameter values $N_{0}=5, R_{0}=5, \rho_{0}=0.2, L_{0}=50, N_{1}=10$, $R_{1}=15, \rho_{1}=0.4$, and $L_{1}=100$, Figure 9.6 shows that if the capacity of buffer $B_{1}$ is small, namely $Q_{1}=20$, then the VBRnr buffer behavior is not well represented by the simpler model. However, if the capacity of $B_{1}$ is substantially larger, e.g., $Q_{1}=400$ as assumed in Figure 9.7, then for the same traffic parameter values of Figure 9.6, we see that the results derived from the one-buffer model agree quite closely with the results of the exact algorithm.

The validity of this approach is likewise confirmed in Figure 9.8, where $N_{0}=45, R_{0}=15, \rho_{0}=0.05, L_{0}=200, N_{1}=60 R_{1}=30, \rho_{1}=0.075$, and $L_{1}=200$. In this instance, the required bandwidth for the GB sources is greater than that of the channel capacity (hypothesis H2), i.e., the comparison here is with the approximate solution.

Finally, we present a few results concerning the transient behavior of the low-priority buffer. Specifically, we are interested in how this buffer 




Figure 9.6 Comparison with the approximate 1-buffer model; $Q_{1}=20$.

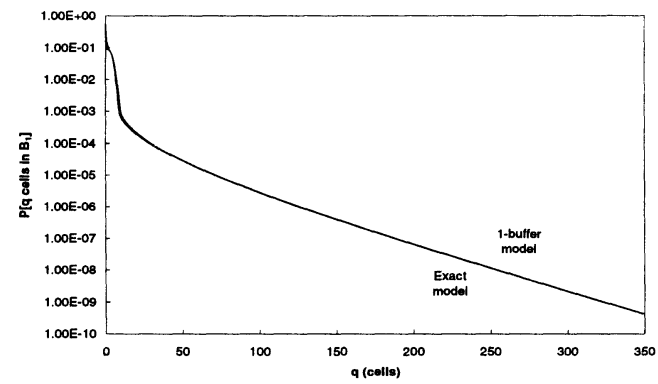

Figure 9.7 Comparison with the approximate 1-buffer model; $Q_{1}=400$.

reacts when another high-priority connection is added to the existing traffic, as motivated by the following concerns.

1) An analysis of steady-state performance, as undertaken above, does not reveal what occurs just after a state-change in the (composite) traffic model.

2) More precisely, when a type- 0 source becomes active (On) at a certain time or, alternatively, when a new source is added in its active state at a certain time, the occupancy probability of the lowpriority buffer gets conditioned by that event, resulting in transient behavior that deserves investigation. 


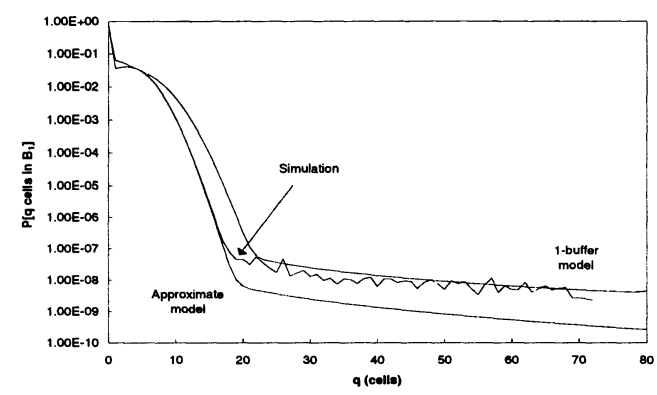

Figure 9.8 Comparison with the approximate 1-buffer model, assuming statistical allocation.

3) Moreover, differences between transient and steady-state performance are likely to be more pronounced as the burstiness of the sources is increased.

As the consequence of a brief study in this regard, we find that 3) indeed holds, where the sources assumed (prior to the transient phase) are as follows.

High priority (Type 0): $N_{0}=4, R_{0}=5, L_{0}=50,100, \rho_{0}=0.33$.

Low priority (Type 1): $N_{1}=8, R_{1}=15, L_{1}=100, \rho_{1}=0.75$.

The system in question is as depicted in Figure 9.1, i.e., there are separate buffers for type- 0 and type- 1 traffic. Given steady-state operation in the presence of the above-stated traffic, a fifth type- 0 source (identical to the other four) is then added at some time $t_{\mathrm{a}}$, where this source is presumed to be active $(\mathrm{On})$ at the time of its addition. Figure 9.9 illustrates the transient behavior of the low-priority buffer for this example, where time is measured relative to $t_{\mathrm{a}}$, i.e., $\tau=t-t_{\mathrm{a}}$, with $\tau \in[0,4000]$. The vertical axis is the congestion probability $P_{\text {full }}(\tau)$, i.e., the probability that buffer $B_{1}$ is full just after the next type-1-arrival slot that follows time $\tau$. We considered this value since it is easy to compute and is a good (qualitative) indicator of the type-1 cell loss probability. The two curves of Figure 9.9 correspond to the two choices of the mean burst length $L_{0}$ indicated above, where we see that greater burstiness ( $L_{0}=100$ as compared with $\left.L_{0}=50\right)$ implies a higher value of $P_{\text {full }}(\tau)$ throughout the transient region. Moreover, as anticipated, these curves increase from the steady-state value at time $t_{\mathrm{a}}$ to the new steady-state 
value in the presence of five type- 0 connections. However, with $L_{0}=50$ the curve is monotone increasing, but with $L_{0}=100$ it is not.

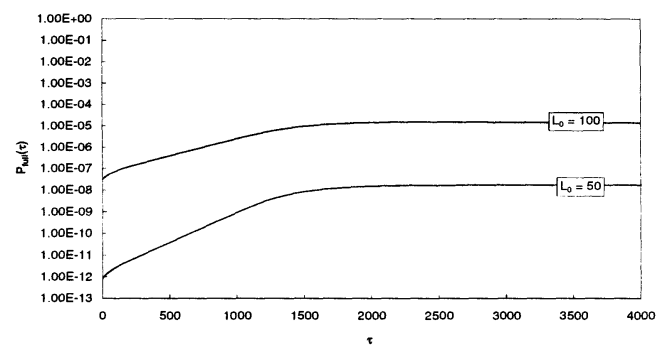

Figure 9.9 Transient congestion probability as a function of $\tau$.

Since this difference is barely perceptible in Figure 9.9, it can be seen more clearly by considering the relative difference

$$
D(\tau)=\frac{P_{\text {full }}(4000)-P_{\text {full }}(\tau)}{P_{\text {full }}(4000)}
$$

and comparing the values for $L_{0}=50$ and $L_{0}=100$ (again for $\tau \in$ $[0,4000])$. This is done in Figure 9.10, where we observe that the curve for $L_{0}=100$ is negative for approximately 2000 time slots, saying that $P_{\text {full }}(\tau)>P_{\text {full }}(4000)$ during this period. This is the most important consequence of the analysis just described. Specifically, beyond what was anticipated in item 3) above, we find that, with sufficiently bursty sources, type-1 cell-loss probabilities during a transient period can exceed those experienced under steady-state conditions. A more thorough investigation is required to assess the magnitude and impact of such differences for various traffic scenarios.

\section{SUMMARY}

This investigation has concerned an ATM multiplexer that is designed to accommodate both GB and VBRnr connections. Service is prioritized via buffers dedicated to each service class, where the low-priority VBRnr traffic is served only if there are no cells in the buffer for the GB connections. The ensuing study then developed an appropriate model for the two-buffer system, followed by a steady-state analysis of how the burstiness of high-priority sources affects the occupancy of the low-priority 


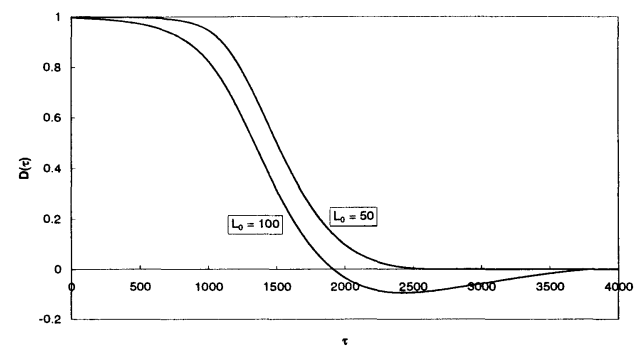

Figure 9.10 Relative difference in transient congestion probability.

buffer under alternative traffic assumptions. The first presumes that the GB connections are allocated according to their peak bit rates; in this case, the analysis was exact. The second presumes partial statistical multiplexing of the GB traffic; here, the exact method was extended so as to provide an approximate solution. The results obtained in this regard reveal that the influence of GB-source burstiness is considerable, even with peak allocation. In cases where the low-priority buffer is sufficiently large (e.g., a capacity of 400), it was shown further that an approximate single-buffer model provides results that conform closely with those obtained by the earlier solution methods (for either allocation hypothesis). Finally, returning to the two-buffer model, its transient behavior was examined in a region that follows the addition of an active high-priority source. Here it was found that, with sufficiently bursty GB traffic, the congestion probabilities for the low-priority buffer can exceed those experienced under steady-state conditions.

\section{References}

Acampora, A. and Zhang, Z. (1992). Effect of ON/OFF distributions on the cell loss probability in ATM networks. In Proc. GLOBECOM'92, pages 1533-1539.

ATM Forum (1996). The ATM Forum traffic management specification Version 4.0. ATM Forum Contribution AFTM 96-0056.000.

Bonomi, F. and Fendick, K. (1995). The rate based flow control framework for the available bit rate ATM service. IEEE Networks.

Bonomi, F., Montagna, S., and Paglino, R. (1992). Busy period analysis for an ATM switching element output line. In Proc. IEEE INFOCOM'g2, pages 544-550. 
F.Bonomi, Fratta, L., Montagna, S., and Paglino, R. (1990). Priority on cell service and on cell loss in ATM switching. In Proc.7th ITC Seminar, Morristown, NJ.

Gopal, I., Guerin, R., Janniello, J., and Theoharakis, V. (1992). ATM support in a transparent network. In Proc. GLOBECOM'g2, pages 301-307.

Jacobsen, S. B., Dittmann, L., Moth, K., and Sallberg, K. (1990). Load control in ATM networks. In Proc. R1012 BLNT RACE Workshop, Monte Carlo, Monaco.

Kroner, H., Hebuterne, G., Boyer, P., and Gravey, A. (1991). Priority management in ATM switching nodes. IEEE J. Select. Areas Commun., pages 418-427.

Meyer, J. F., Montagna, S., and Paglino, R. (1993). Dimensioning of an ATM switch with shared buffer and threshold priority. Computer Networks and ISDN Systems, pages 95-108.

Rasmussen, C., Sorensen, J. H., Kvols, K. S., and Jacobsen, S. B. (1991). Source-independent call acceptance procedures in ATM networks. IEEE J. Select. Areas of Commun., pages 351-356.

Sohraby, K. (1992). Heavy traffic multiplexing behavior of highly bursty heterogeneous sources and their admission control in high speed networks. In Proc. GLOBECOM'92, pages 1518-1523.

Zhang, J. (1993). Performance study of Markov modulated fluid flow models with priority traffic. In Proc. IEEE INFOCOM'93, pages 1017. 